\title{
A Case of Body Packing: Internal Smuggling of Illicit Drugs
}

Michael A. Valentino, MD, PhD

The patient is a 27-year old Hispanic female who was brought to the ER from the airport by the Drug Enforcement Agency (DEA) on suspicion of "body packing" (illicit drug smuggling via ingestion). An abdominal X-ray (Figures 1a,b) was obtained in the ER which showed multiple radiopaque foreign bodies which were uniform in size measuring 5 $\mathrm{cm} \times 2 \mathrm{~cm}$ throughout her colon. The patient then admitted to ingesting numerous heroin pellets. She subsequently had a CT Abdomen/Pelvis performed (Figure 2) which again showed numerous tubular radiopaque foreign bodies throughout the gastrointestinal tract. She was admitted to the medical intensive care unit (MICU) under police escort. She was treated with oral laxatives to accelerate passage of the pellets, and she was monitored for signs of opiate intoxication due to pellet rupture. Surgery was consulted in the event of pellet rupture and resultant drug toxicity uncontrolled with pharmacologic therapy as well other potential complications including intestinal obstruction and/ or perforation. She had serial abdominal x-rays performed to monitor for passage of all of the heroin pellets. All heroin pellets were collected by the DEA as evidence. During her hospitalization, the patient was able to pass all of the heroin pellets without any adverse events and was discharged into police custody.
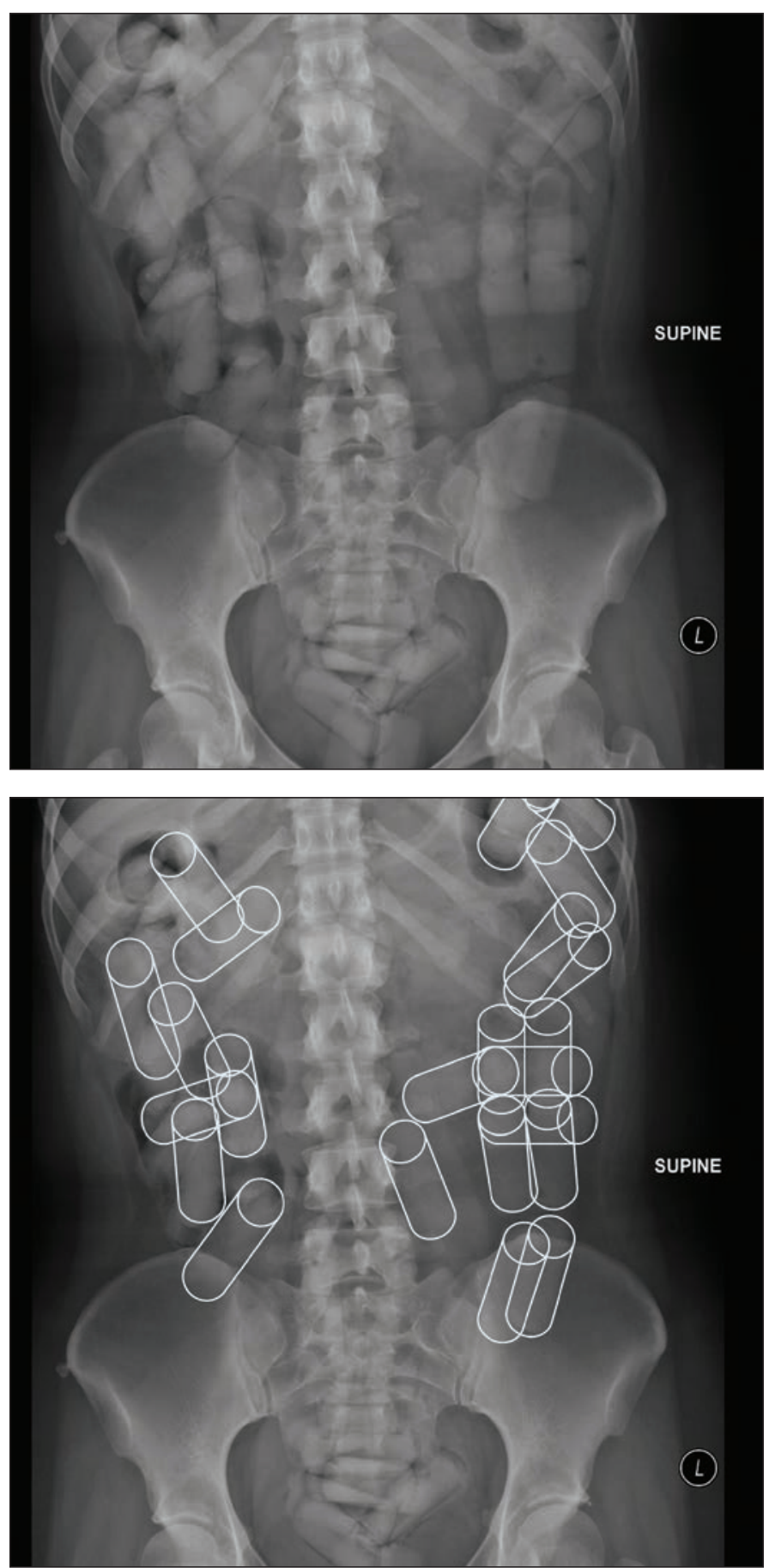


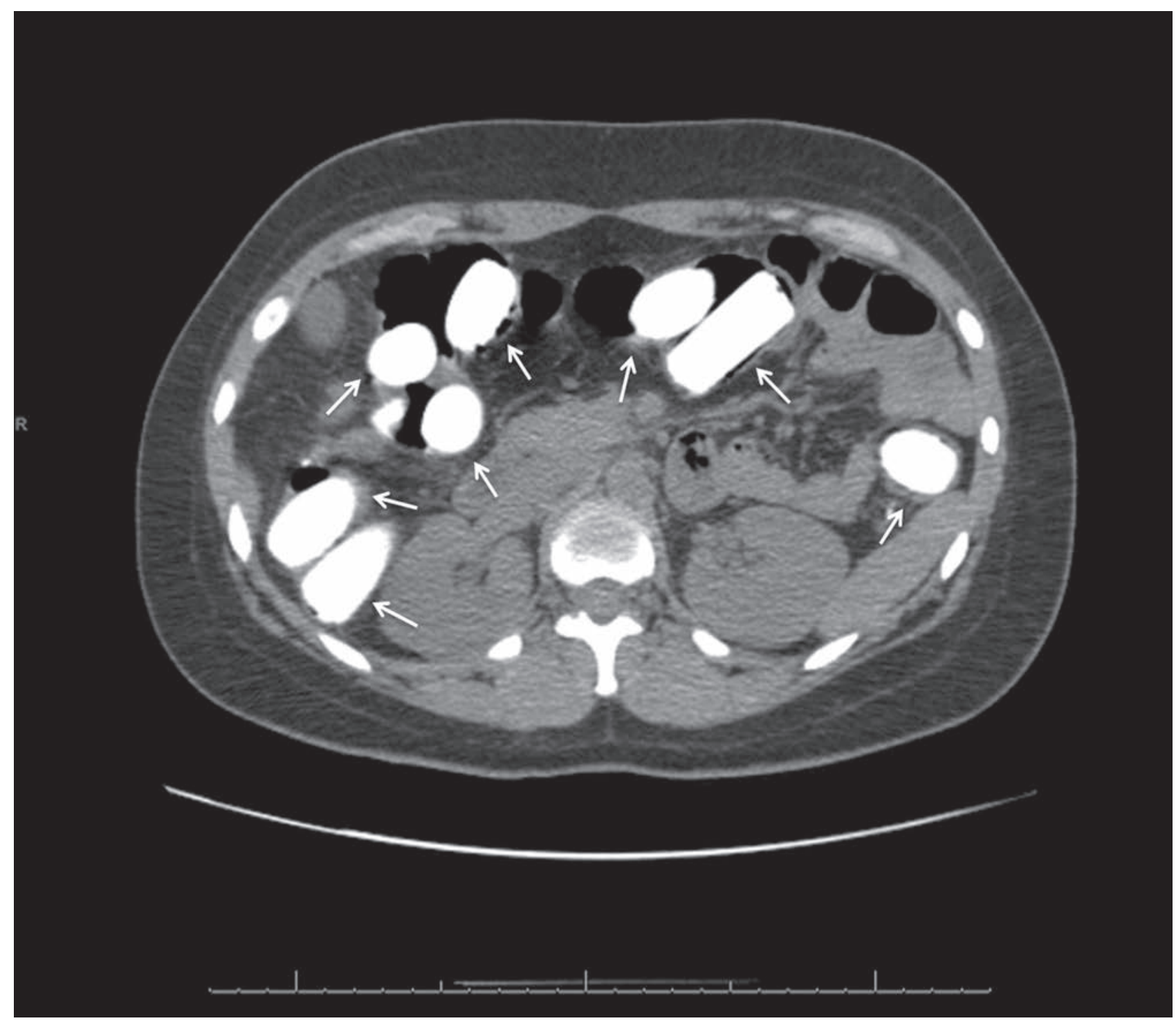

Figure 2. Admission CT Abdomen/Pelvis

The Medicine Forum | 65 